\title{
Sound can improve visual search in developmental dyslexia
}

\author{
Liselotte de Boer-Schellekens · Jean Vroomen
}

Received: 22 August 2011/Accepted: 23 October 2011/Published online: 8 November 2011

(C) The Author(s) 2011. This article is published with open access at Springerlink.com

\begin{abstract}
We examined whether developmental dyslexic adults suffer from sluggish attentional shifting (SAS; Hari and Renvall in Trends Cogn Sci 5:525-532, 2001) by measuring their shifting of attention in a visual search task with dynamic cluttered displays (Van der Burg et al. in J Exp Psychol Human 34:1053-1065, 2008). Dyslexics were generally slower than normal readers in searching a horizontal or vertical target among oblique distracters. However, the addition of a click sound presented in synchrony with a color change of the target drastically improved their performance up to the level of the normal readers. These results are in line with the idea that developmental dyslexics have specific problems in disengaging attention from the current fixation, and that the phasic alerting by a sound can compensate for this deficit.
\end{abstract}

Keywords Dyslexia · Visual search task .

Visual attention - Orienting of attention

\section{Introduction}

Developmental dyslexia is a neurobiological disorder characterized by a difficulty in reading acquisition despite adequate intelligence, conventional education, and motivation (APA 1994). The prevailing view supports the hypothesis that dyslexia results from a specific deficit of auditory-phonological perception, representation, and phonological memory (see Vellutino et al. 2004; Ziegler

L. de Boer-Schellekens · J. Vroomen ( $₫)$

Department of Psychology, Tilburg University,

P.O. Box 90153, 5000 LE Tilburg, The Netherlands

e-mail: J.Vroomen@uvt.nl and Goswami 2005 for reviews). Children and adults with dyslexia show, indeed, deficits in the representation and manipulation of phonological information (e.g., poor speech-sound awareness, slow lexical retrieval and poor phonological short-term memory; see Ramus 2003, for a review). These deficits could interfere with one of the most critical skills for successful reading acquisition, such as phonological decoding (Share 1995; Ziegler and Goswami 2005).

Apart from their phonological difficulties, dyslexic subjects often suffer from a variety of subtle sensory and motor deficits. Whether these deficits have any causal relation to the reading disorder, or are totally independent, is currently under debate. One hypothesis of a visual cause for dyslexia is that the reading disorder is caused by a deficiency in the magnocellular part (also referred to as the "transient system") of the visual system (Stein and Walsh 1997). This hypothesis might seem controversial, since one would expect the parvo- rather than the magnocellular pathways to be largely involved with fine pattern vision and object discrimination that are essential for reading. However, studies have indicated a function for the magnocellular-dominated dorsal stream in selective attention (Motter 1993; Vidyasagar 1998).Vidyasagar and Pammer (1999) suggested that if the magnocellular system (M system) is involved in gating all visual input going through the striate cortex, a deficit in this system would also affect the parvocellular system (P system). This would become manifest in tasks in which there is intense competition for attentional resources and in which the supposed M-mediated attentional spotlight is essential for good performance. Stein (2003) also suggested that a magnocellular deficiency may cause a type of visual attention deficit in dyslexia. This has made it important to assess visual attention in dyslexic readers in more detail. A recent and particularly 
interesting hypothesis that links the magnocellular deficit with reading problems is that dyslexics may have "sluggish attentional shifting" (SAS) (Hari and Renvall 2001).

The present study was motivated by the idea that SAS could indeed provide a coherent framework to understand a variety of sensory problems that dyslexics encounter. The basic notion underlying SAS is that sensory input is chunked and that attention of dyslexic subjects, once engaged on a chunk, cannot be easily disengaged (Hari and Renvall 2001). This causes impairments in the shift or the focusing of attention (Hari and Renvall 2001), and it may result in a prolonged attentional dwell time and poor judgments of temporal order (Jáskowski and Rusiak 2008). SAS can affect fluent reading because the sensory input is prolonged, thereby degrading essential cortical representations (for review, see Hari and Renvall 2001). In line with this idea, Lallier et al. (2010) used an auditory and visual stream segregation task and reported that in order to process two successive stimuli separately, dyslexic participants with phonological impairments required a significantly longer inter-stimulus interval than controls regardless of sensory modality. Another important prediction from SAS is that dyslexics may profit from a transient sound because of a general alerting effect that improves the disengagement of attention.

A number of methods have been used to measure visual attention in dyslexics, but so far, none has used sounds to improve search time. In the standard visual search task, participants search for a prespecified visual target among other distracters. Previous reports are rather consistent with a visual attention deficit in dyslexics, reporting slower search times for dyslexic than normal readers (Williams et al. 1987; Ruddock 1991; Casco and Prunetti 1996; Vidyasagar and Pammer 1999; Sireteanu et al. 2008; Romani et al. 2011). In this context, Pammer and Vidyasagar (2005) and Jones et al. (2008) argued that dyslexics may suffer from an impairment in the serial allocation of attention. According to SAS, the slowness of dyslexics can be explained by an impairment in the disengagement of attention from an element in the search set.

Here we thought to add sounds to the visual search task, using the "pip-and-pop" paradigm by Van der Burg et al. (2008). These authors designed a visual search task in which a target (a horizontal or vertical line) was embedded in a cluttered display of distracters (oblique lines). The targets and distracters changed, on randomly determined times color (and, important from the perspective of the magnocellular system, also luminance) from green-to-red or red-to-green. They found that a simple auditory pip could drastically decrease search times if the pip was synchronized with the color/luminance-change of the target: the "pip" then made the target "pop-out." Further studies have shown that a sound will only lead to benefits in visual search if the changes in the two signals are both synchronized and transient (Van der Burg et al. 2010). If this condition is met, then the effect will also resist wide spatial misalignment (Fiebelkorn et al. 2011).

In the current study, we used this paradigm to examine whether a sound would improve the visual search time of dyslexic readers more than it does in normal readers. If, as proposed in SAS, dyslexic readers have problems with the disengagement of attention from the current fixation, one expects them in the tone-absent condition (serial search) to have longer search times than normal readers, and their slope of the search time per item should be steeper (see also Vidyasagar and Pammer 1999; Sireteanu et al. 2008; Romani et al. 2011). In the sound-present condition, though, the pip can make the target pop-out (parallel search), and search time may become independent of the set size of the distracters. This should be particularly helpful for dyslexic readers, as they may have a specific difficulty with serial, but not parallel, search (Sireteanu et al. 2008). Ultimately, then, a single pip may compensate for the dyslexics' visual attention deficit.

\section{Method}

\section{Participants}

Fifteen young adults with developmental dyslexia (five men and ten women) and 15 age-matched controls without reading difficulties (five men, ten women) were tested. The dyslexic readers had been diagnosed with developmental dyslexia based on standard exclusion criteria (APA 1994). They were all formally assessed and diagnosed by clinical and educational psychologists. Their reading achievements (accuracy and/or speed) were additionally assessed via standardized Dutch reading tests for single word and nonword reading (Brus and Voeten 1997; Van den Bos et al. 1999). Dyslexic participants were selected on the basis of (1) normal or corrected-to-normal vision and hearing; (2) absence of neurological and/or psychiatric disorders; (3) absence of attention deficit disorder with hyperactivity (because of the high comorbidity with dyslexia); (4) absence of color blindness. The controls reported no history of reading problems. The two groups were drawn from the same subject pool of university students, but were significantly different for both accuracy and speed of word and nonword reading (see Table 1 for details). Participants were tested individually, were unaware of the purpose of the experiment, and received course credits or money for their participation. Written consent was obtained from all participants according to the Declaration of Helsinki. 
Table 1 Mean and standard deviation (SD) of age (in years), word and nonword reading scores (errors and speed in number of correctly read items) in dyslexics $(N=15)$ and agematched normal reading controls $(N=15)$

The bold values are with $P<0.05$

\begin{tabular}{|c|c|c|c|c|c|c|}
\hline & \multicolumn{2}{|c|}{ Dyslexics } & \multicolumn{2}{|c|}{ Controls } & \multicolumn{2}{|c|}{ Comparison } \\
\hline & Mean & SD & Mean & SD & $t(28)$ & $P$ \\
\hline Age & 21.5 & 2.2 & 20.7 & 1.7 & -1.1 & 0.28 \\
\hline \multicolumn{7}{|c|}{ Words reading } \\
\hline Errors & 1.3 & 1.1 & 0.3 & 0.6 & -3.09 & 0.004 \\
\hline Speed & 77.8 & 10.3 & 93.5 & 16.4 & 3.16 & 0.004 \\
\hline \multicolumn{7}{|c|}{ Nonword reading } \\
\hline Errors & 8.9 & 3.0 & 2.3 & 2.9 & -6.73 & $<0.001$ \\
\hline Speed & 69.9 & 16.2 & 98.5 & 14.0 & 5.17 & $<0.001$ \\
\hline
\end{tabular}

Stimuli

The stimuli were made as in Van der Burg et al. (2008). The auditory stimulus was a short white noise click of $68 \mathrm{~ms}$ presented at $74 \mathrm{~dB}(\mathrm{~A})$ through the laptop speakers. The visual stimuli were presented on a $15-\mathrm{inch}, 60-\mathrm{Hz}$ laptop monitor (Dell Latitude E5500), controlled by E-Prime 1.2 (Psychology Software Tools, Inc.; http://www.pstnet.com/ eprime). The visual search displays consisted of 24 or 48 red $\left(20 \mathrm{~cd} \mathrm{~m}^{-2}\right)$ and green $\left(11 \mathrm{~cd} \mathrm{~m}^{-2}\right)$ line segments (length $0.88^{\circ}$ visual angle) against a dark background $\left(0.05 \mathrm{~cd} \mathrm{~m}^{-2}\right)$. The initial color (red or green) was randomly determined for each item. The lines were randomly placed in an invisible $10 \times 10$ grid $\left(10.5^{\circ} \times 6.5^{\circ}\right)$ centered on a white central fixation cross, with the constraint that the target was never presented at the four central positions, to avoid immediate detection. The target was a horizontal or vertical line, while for distracters line orientation deviated randomly by plus or minus $26.5^{\circ}$ from horizontal or vertical. The distracters changed color (from red-to-green or vice versa) every 50,100 , or $150 \mathrm{~ms}$. The number of distracters that changed simultaneously during a trial varied for the different set sizes; in set size $24,1,2$ or 4 distracters changed simultaneously, while in set size 48 , it was 1,4 or 7 distracters. The target changed color every 500 or $1,000 \mathrm{~ms}$, and always changed alone. Distracters did not change color from $150 \mathrm{~ms}$ before the target until $100 \mathrm{~ms}$ after the target had changed color. During the first $500 \mathrm{~ms}$ of a trial, the target also did not change color. A dynamic example of trials with and without sound can be found at http://www.psy.vu.nl/pippop/.

\section{Procedure}

Participants were tested in a dimly lit and sound-proof cabin and were seated approximately $65 \mathrm{~cm}$ in front of the laptop screen. Head movements were precluded by a chinrest. A white fixation cross was illuminated in the center of the screen at the beginning of each trial. Participants were asked to remain focused on the fixation cross. After
150-500 ms, the display with target and distracters appeared at the screen. In the sound-present condition, a change in the color of the target was always accompanied by a simultaneously presented sound. The search display was presented until the participants made a response. Participants were instructed to search for the target and to press one of two buttons corresponding with the target orientation "-" or "l" as fast and accurately as possible. All participants were explicitly told that sounds, if present, were synced with a color change of the target, and that they thus could benefit from the sound because it signaled that the target had changed color. To encourage that participants reacted as fast and as accurately as possibly throughout the whole experiment, written feedback about accuracy and search time was given after each trial. Overall scores were also given at the end of the experiment. A practice session preceded the experimental test that stopped until 10 consecutively correct answers were given.

Design

There were two within-subject factors: set size ( 24 or 48 ) and sound (present or absent). These factors were varied randomly across trials. Target orientation was balanced and randomly mixed. The whole test consisted of 1 block of 80 experimental trials, in which each of the 4 unique conditions was presented 20 times. The experiment lasted $\sim 15$ min in total.

\section{Results}

The data of the practice session and erroneous responses were excluded from analyses. The overall mean error rate was low $(5.8 \%$ for the dyslexic group and $4.4 \%$ for the control group) and did not significantly differ between groups, $t(28)=1.32, P>0.05$. No further analyses were therefore performed on error rates. Search time was measured from the onset of the search display until the response to the target. The averaged search times for each 
condition are presented in Fig. 1. An overall repeatedmeasures ANOVA was conducted with group (dyslexics versus normal readers) as between-subject factor, and set size (24 or 48 items) and sound (sound present or absent) as within-subject factors. As expected, dyslexics had longer overall search times than normal readers, $F(1,28)=5.66$, $P<0.05, \eta \mathrm{p} 2=0.17$ (mean search time of $8,004 \mathrm{~ms}$ for dyslexics and 6,100 $\mathrm{ms}$ for normal readers). The search time was also faster for the small than large set size, $F(1,28)=144.94, P<0.001, \eta \mathrm{p} 2=0.84$ (mean search time of 4,759 ms for set size 24 and 9,351 ms for set size 48 ), and search time of trials with sound was faster than without sound, $F(1,28)=13.58, P<0.01, \eta \mathrm{p} 2=0.17$ (mean search time of 7,929 ms for sound-absent conditions and $6,181 \mathrm{~ms}$ for sound-present conditions). There was an interaction between set size and group, $F(1,28)=7.15$, $P<0.05, \eta \mathrm{p} 2=0.20$, indicating that dyslexics had slower search times per item than normal readers (dyslexics: $222 \mathrm{~ms} /$ item; normal readers: $170 \mathrm{~ms} / \mathrm{item}$ ). Most importantly, there was interaction between group, sound, and set size, $F(1,28)=5.275, P<0.05, \eta \mathrm{p} 2=0.16$. As is clearly visible in Fig. 1, and as predicted, both groups benefitted from sound, but the dyslexics profited more from sound than normal readers as their improvement in search times per set size was bigger (an improvement of 1,000 ms for set size 24 , and $4,735 \mathrm{~ms}$ for set size 48 ) than that of the controls (an improvement of $281 \mathrm{~ms}$ for set size 24, and $976 \mathrm{~ms}$ for set size 48).

Separate ANOVAs on the sound-absent and soundpresent conditions showed that in the sound-absent conditions, the dyslexics were significantly slower than the normal readers, $F(1,28)=9.15, P<0.01, \eta \mathrm{p} 2=0.25$, and this difference was bigger with the large set size (set size $\times$ group interaction effect, $F=9.52, \quad P<0.01$, $\eta \mathrm{p} 2=0.25)$. In stark contrast, in the sound-present condition, the search time of the dyslexic group was not
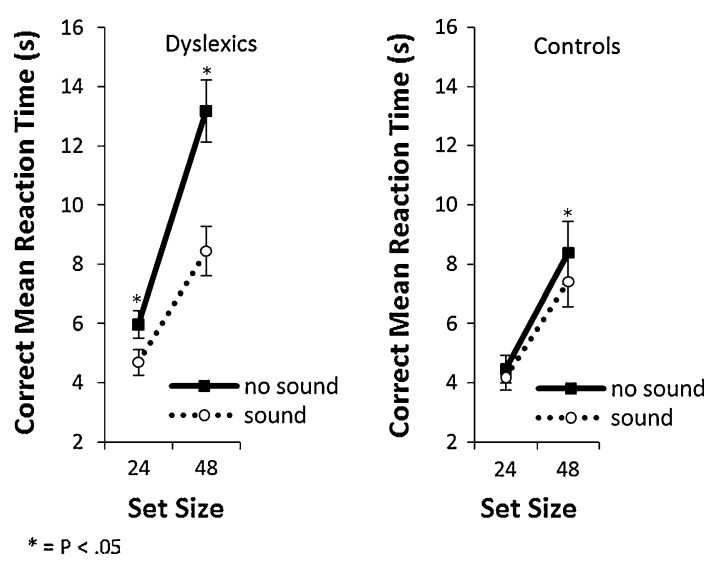

Fig. 1 Mean search time (in seconds) as a function of set size and presence of sound for the dyslexic (left panel) and normal reading (right panel) group. Error bars represent 1 standard error of mean different from the control group (main effect of group, $F<1$, set size $\times$ group, $F<1$ ).

\section{Discussion}

Here we examined the effect of sound on visual search times of dyslexic versus normal readers, using the "pipand-pop" task. Our results confirmed, in accordance with predictions from SAS, that (1) in the sound-absent condition, dyslexics' search time was much longer than of normal readers, (2) in the sound-absent condition, dyslexics' search time increased more strongly with an increase in set size than of normal readers, and (3) dyslexics' search time improved drastically by the presence of a transient sound; in fact their search time then became as good as normal readers.

The general slowness of the dyslexic group is consistent with previous studies using visual search tasks (e.g., Vidyasagar and Pammer 1999; Sireteanu et al. 2008; Romani et al. 2011). These findings are in accordance with the "sluggish attentional shifting" account of Hari and Renvall (2001), according to which the dyslexics' automatic attention system cannot disengage fast enough from one item to move to the next. This results in a prolonged dwell time and large effect of set size.

The improvement in performance by the presence of the sound leads to the question why a transient sound could speed up the orienting of attention of dyslexic readers as much as it does. First of all, it is important to note that the sound used in the experiment did not bias attention toward a specific direction or location. We used a static sound that was centrally presented, so the sound was not informative about the possible location of the target. The gain offered by the sound for dyslexic readers is in line with other findings, demonstrating that dyslexics have specific problems in serial, but not parallel, search. For example, Sireteanu et al. (2008) demonstrated that dyslexics, compared to normal readers, have difficulties in serial, but not parallel, search. Our results are consistent with that (and previous findings by Williams et al. 1987; Ruddock 1991; Casco and Prunetti 1996; Vidyasagar and Pammer 1999) in that we found that in the sound-absent condition, the search time of dyslexic readers increased more with the number of distracters and was significantly slower than that of the normal readers. In the sound-present condition, it has been argued that the binding of synchronized auditory-visual signals occurs rapidly, automatically, and effortlessly, with the auditory signal attaching to the visual signal relatively early in the perceptual process. Through this, a nonspatial auditory event ("pip") can guide attention toward the location of a synchronized visual event that, without an auditory signal, is difficult (Van der Burg et al. 2008). 
The "pip" then makes the target "pop-out," changing a serial search into an automatic, parallel search. This then led to a decrease in dyslexics search times up to the level of normal readers.

Although it is clear that dyslexics were better in parallel search than serial search, one can still ask whether there were-besides SAS-any other reasons why dyslexics benefited significantly more from sound than the normal readers. One clue for this may come from a visual search study by Facoetti et al. (2000). These authors found that dyslexic children had a bigger pop-out effect, which is a characteristic of parallel processing. The authors argued that dyslexia is characterized by a difficulty to narrow the attentional focus, and dyslexics therefore tended to adopt a more distributed focus of attention. In addition, Van der Burg et al. (2008) suggested that at least some distributed attention is necessary for observers to notice the synchronized event of the "pip" and "pop." By combining these two notions, it may become understandable why dyslexics, presumably with a more distributed focus of attention, could profit more from sound than normal readers.

Plausible explanations for the substantial benefit of sound by dyslexics might also be derived from studies that examined the effect of sound on visual attention. Robertson et al. (1998) studied patients with neglect and showed that the phasic alerting by a transient nonspatial sound can overcome their spatial deficits in visual awareness. This finding provides evidence that the phasic alerting alone can directly affect the speed of perceptual processing, rather than merely affecting motor readiness. Follow-up studies by Van der Burg et al. (2008, 2011), however, found evidence for a very early multisensory interaction that ruled out that the pip-and-pop effect is due to increases of alertness, as the effect follows a time course that is quite different from alerting effects.

Doyle and Snowden (2001) also examined the effects of simple auditory signals whose onset was synchronized with that of the visual target, but provided no information about the target location. Their findings made them speculate that an auditory signal may promote attentional disengagement (for similar reasoning, see Keetels and Vroomen (2011)). Related to our study, the sound may thus have a facilitatory effect on the disengagement of attention of the dyslexic readers, making them process the visual target (much) sooner when accompanied by a sound relative to when visual information is presented alone.

A final speculation about the reason for the bigger improvement by sound in the dyslexic group may be related to crossmodal temporal deficits that have been found in dyslexia. As demonstrated by Van der Burg et al. (2008), in order to be effective, a sound needs to be presented in close temporal proximity to the visual target change. This thus demands intact crossmodal temporal integration. Various authors, though, argued that dyslexics exhibit deficits in different sensory systems, which involve alterations in temporal information processing (e.g., Tallal 1980; Kinsbourne et al. 1991; Laasonen et al. 2002). One hypothesis confirmed by Hairston et al. (2005) could be relevant. The authors found support for the idea of altered crossmodal temporal processing in dyslexia, as they reported that dyslexic subjects showed an extended temporal window for binding visual and auditory cues. These findings could be potentially relevant for our results, because an enlarged temporal window may lead to a bigger "pip-and-pop" effect in the dyslexics, as they may profit over an extended period of time of the sound. It should be noted, though, that an enlarged temporal window of integration may also lead to more spurious binding between the sound and color changes of distracters. An enlarged temporal window would then interfere rather than being of help in visual search time. Future studies that vary the SOA between sound and target and the SOA between sound and distractor change might give more detailed information on this and on the mechanisms of multisensory integration in dyslexic readers.

To summarize, our results showed that dyslexic readers have problems with visual serial search. This is evidence for visual attentional abnormalities in dyslexia, more specifically in the disengagement and shifting of attention. Most interestingly, though, a spatially noninformative transient sound could overcome these abnormalities. It remains for future studies with other clinical populations who are thought to have difficulties with disengaging and shifting attention (e.g., high-functioning autistic individuals) to further explore the effects of sound on visual attention.

Open Access This article is distributed under the terms of the Creative Commons Attribution Noncommercial License which permits any noncommercial use, distribution, and reproduction in any medium, provided the original author(s) and source are credited.

\section{References}

APA (1994) Diagnostic and statistical manual of mental disorders, 4th edn. American Psychiatric Association, Washington DC

Brus BT, Voeten MJM (1997) Een-Minuut-Test. Pearson, Amsterdam

Casco C, Prunetti E (1996) Visual search in good and poor readers: effects with single and combined features targets. Percept Motor Skills 82:1155-1167

Doyle MC, Snowden RJ (2001) Identification of visual stimuli is improved by accompanying auditory stimuli: the role of eye movements and sound location. Perception 30:795-810

Facoetti A, Paganoni P, Lorusso ML (2000) The spatial distribution of visual attention in developmental dyslexia. Exp Brain Res 132:531-538

Fiebelkorn IC, Foxe JJ, Butler JS, Molholm S (2011) Auditory facilitation of visual-target detection persists regardless of retinal eccentricity and despite wide audiovisual misalignments. Exp Brain Res 213:167-174 
Hairston WD, Burdette JH, Flowers DL, Wood FB, Wallace MT (2005) Altered temporal profile of visual-auditory multisensory interactions in dyslexia. Exp Brain Res 166:474-480

Hari R, Renvall H (2001) Impaired processing of rapid stimulus sequences in dyslexia. Trends Cogn Sci 5:525-532

Jáskowski P, Rusiak P (2008) Temporal order judgment in dyslexia. Psychol Res 72:65-73

Jones MW, Branigan HP, Kelly ML (2008) Visual deficits in developmental dyslexia: relationships between non-linguistic visual tasks and their contribution to components of reading. Dyslexia 14:95-115

Keetels M, Vroomen J (2011) Sound affects the speed of visual processing. J Exp Psychol Human 37:699-708

Kinsbourne M, Rufo DT, Gamzu E, Palmer RL, Berliner AK (1991) Neurological deficits in adults with dyslexia. Dev Med Child Neurol 33:763-775

Laasonen M, Service E, Virsu V (2002) Crossmodal temporal order and processing acuity in developmentally dyslexic young adults. Brain Lang 80:340-354

Lallier M, Tainturier M-J, Dering B, Donnadieu S, Valdois S, Thierry G (2010) Behavioral and ERP evidence for amodal sluggish attentional shifting in developmental dyslexia. Neuropsychologia 48:4125-4135

Motter BC (1993) Focal attention produces spatially selective processing in visual cortical areas $\mathrm{V} 1, \mathrm{~V} 2$, and $\mathrm{V} 4$ in the presence of competing stimuli. J Neurophysiol 70:909-919

Pammer K, Vidyasagar TR (2005) Integration of the visual and auditory networks in dyslexia: a theoretical perspective. J Res Read 28:320-331

Ramus F (2003) Developmental dyslexia: specific phonological deficit or general sensorimotor dysfunction? Curr Opin Neurobiol 13:212-218

Robertson IH, Mattingley JB, Rorden C, Driver J (1998) Phasic alerting of neglect patients overcomes their spatial deficit in visual awareness. Nature 395:169-172

Romani C, Tsouknida E, di Betta AM, Olson A (2011) Reduced attentional capacity, but normal processing speed and shifting of attention in developmental dyslexia: evidence from a serial task. Cortex 47:715-733

Ruddock KH (1991) Visual search and dyslexia. In: Stein JF (ed) Vision and visual dyslexia. MacMillan Press, London, pp 58-79
Share DL (1995) Phonological recoding and self-teaching- sine- quanon of reading acquisition. Cognition 55:151-218

Sireteanu R, Goebel C, Goertz R, Werner I, Nalewajko M, Thiel A (2008) Impaired serial visual search in children with developmental dyslexia. In: Eden GF, Flower DL (eds) Learning, skill acquisition, reading, and dyslexia, vol 1145. Blackwell, Oxford, pp 199-211

Stein J (2003) Visual motion sensitivity and reading. Neuropsychologia 41:1785-1793

Stein J, Walsh V (1997) To see but not to read; the magnocellular theory of dyslexia. Trends Neurosci 20:147-152

Tallal P (1980) Auditory temporal perception, phonics and reading disabilities in children. Brain Lang 9:182-198

Van den Bos KP, Lutje Spelberg HC, Scheepsma AJM, De Vries JR (1999) De Klepel: pseudowoordentest. Harcourt Test Publishers, Amsterdam

Van der Burg E, Olivers CNL, Bronkhorst AW, Theeuwes J (2008) Pip and pop: nonspatial auditory signals improve spatial visual search. J Exp Psychol Human 34:1053-1065

Van der Burg E, Cass J, Olivers CNL, Theeuwes J, Alais D (2010) Efficient visual search from synchronized auditory signals requires transient audiovisual events. Plos One 5:11. e10664

Van der Burg E, Talsma D, Olivers CNL, Hickey C, Theeuwes J (2011) Early multisensory interactions affect the competition among multiple visual objects. Neuroimage 55:1208-1218

Vellutino FR, Fletcher JM, Snowling MJ, Scanlon DM (2004) Specific reading disability (dyslexia): what have we learned in the past four decades? J Child Psychol 45:2-40

Vidyasagar TR (1998) Gating of neuronal responses in macaque primary visual cortex by an attentional spotlight. Neuroreport 9:1947-1952

Vidyasagar TR, Pammer K (1999) Impaired visual search in dyslexia relates to the role of the magnocellular pathway in attention. NeuroReport 10:1283-1287

Williams MC, Brannan JR, Latirgue EK (1987) Visual search in good and poor readers. Clin Vision Sci 1:367-371

Ziegler JC, Goswami U (2005) Reading acquisition, developmental dyslexia, and skilled reading across languages: a psycholinguistic grain size theory. Psychol Bull 131:3-29 